\title{
Effect of Direct Transcranial Stimulation by Continuous Current (Tdcs) in "Kiss Nightclub Fire" Patients with Post-Traumatic Stress Disorder: Phase II Controlled Clinical Trial
}

\section{Kathy Aleixo dos Santos Marcolin}

Univeristy of Santa Maria (UFSM) - Santa Maria City - Rio Grande do Sul State

Ângelo Batista Miralha Cunha

Univeristy of Santa Maria (UFSM) - Santa Maria City - Rio Grande do Sul State

\section{Beatriz Capparros Yoneyama}

Univeristy of Santa Maria (UFSM) - Santa Maria City - Rio Grande do Sul State

Tiango Aguiar Ribeiro ( $\sim$ tiangoribeiro@gmail.com )

Univeristy of Santa Maria (UFSM) - Santa Maria City - Rio Grande do Sul State

\section{Research Article}

Keywords: post-traumatic stress disorder, transcranial direct current stimulation, depression, anxiety.

Posted Date: December 16th, 2021

DOI: https://doi.org/10.21203/rs.3.rs-987110/v1

License: (c) (1) This work is licensed under a Creative Commons Attribution 4.0 International License.

Read Full License 


\section{Abstract}

\section{Background}

Victims of disasters can develop post-traumatic stress disorder (PTSD) in 30-40\% of cases. Application of repetitive transcranial magnetic stimulation (rTMS) shown promising results in PTDS. Transcranial direct current stimulation (tDCS) similar to rTMS, which is a neuromodulation technique, has shown promise in treatment of neuropsychiatric disorders.

Method

A clinical trial conducted from March/2015-July-2016 in "KISS nightclub fire" disaster patients diagnosed with PTSD without complete remission of symptoms, over 18-years and who maintained pharmacological treatment. Electrodes positioned as cathode (right DLPFC) and extra-cephalic anode (contralateral deltoid muscle.) A current of $2 \mathrm{~mA}$ used for $25 \mathrm{~cm}^{2}$ area $\left(0.08 \mathrm{~mA} / \mathrm{cm}^{2}\right.$ current density) $30 \mathrm{~min}$ once a day, for 10 continuous days. Patients were assessed pre-intervention, post-intervention, 30days' and 90-days' post-intervention. PTSD Checklist-Civilian version (PCL-C); Montreal Cognitive Assessment (MoCA); Hamilton Depression and Anxiety Rating Scale (HAM-D and HAM-A) were used.

Results

145 subjects were initially screened and eight analysed. $87,5 \%$ were female. $30.88 \pm 7.74$ years were the mean age (ranging 23-44). post-intervention results demonstrate: no cognitive impairment (MoCA); $60 \%$ reduction in HAM-D scale (patients diagnose as moderate depression turns normal diagnosis) $(p<0.001)$; $54.39 \%$ reduction in HAM-A scale (moderate to severe symptoms of anxiety patients turns mild symptoms) $(p<0.001)$ and $20 \%$ decrease in PCL-C scale values (patients with high severity symptoms of PTSD turns moderate to moderately high severity symptoms) $(p<0.001)$. Improvement in PTSD symptoms were maintained 30-days post-intervention ( $P C L-C, p=0.025)$ and improvement in symptoms of depression (HAM-D, $p=0.006)$ and anxiety (HAM-A, $p=0.028)$ in 90-days post-intervention.

\section{Conclusion}

Despite decrease over time, this improvement in PTSD, depression and anxiety symptoms was maintained throughout the first month after treatment. tDCS adjuvant can be an alternative treatment to refractory PTSD, either as a monotherapy or as a treatment enhancement strategy. They can also be an option for patients who do not want or do not tolerate pharmacological management.

RBR-2qpv74b, 01/07/2021, "retrospectively registered" 03/03/2015.

\section{Background}

Post-traumatic stress disorder (PTSD) is a syndrome triggered after exposure to a traumatic event. As diagnostic criteria the presence of several symptoms is observed, such as intrusive recall of aspects of 
the event, avoidance of reminders, anger, hyper-vigilance, dysphoria, sleeplessness, increased arousal or anhedonia (1-4). About $60.7 \%$ of men and $51.2 \%$ of women will be exposed to at least one potentially traumatic event during their lives (5). The prevalence of PTSD during life in the general population of the United States is $8 \%$ (6) but in direct victims of disasters these numbers can reach $30-40 \%$ (7). Most PTSD patients have severe distress and some impairment in their psychosocial functioning, such as family and work conflicts, and/or the development of comorbidities (social phobia, panic syndrome, major depressive disorder, increased risk of suicide) $(1,6,8)$.

Treatment includes psychotherapy and cognitive behavioral therapies such as prolonged exposure therapy, cognitive processing therapy, eye movement desensitization and reprocessing cognitive behavioral therapy $(2,9)$. However, pharmacological therapy also has its place, the first-line treatment being the use of antidepressants, particularly selective serotonin reuptake inhibitors (9); the best responses are seen with paroxetine and sertraline (10). However, despite the correct treatment, less than $60 \%$ of patients respond to treatment and only $30 \%$ have complete remission of symptoms (11).

A large fire in a nightclub in Santa Maria, Rio Grande do Sul, Brazil, in January 2013, killed 242 young people, left hundreds more with physical and psychological consequences and was the biggest disaster

to date in our country. The event, known in the media as "KISS nightclub fire", caused an increase in the number of cases of several pathologies, among them PTSD. The impact of this disease, not only on KISS survivors but also on family members, first responders and police, combined with the low effectiveness of standard treatment, made new forms of approach necessary.

In this context, transcranial direct current stimulation (tDCS), which is a neuromodulation technique, has shown promise in the treatment of neuropsychiatric disorders (12-14). tDCS consists of the passage of low-intensity constant electric current through the skull, thus modifying brain activity $(12,14,15)$. The application of repetitive transcranial magnetic stimulation (rTMS), a technique similar to tDCS, has shown promising results in the treatment of patients with PTSD. Several studies with low-frequency rTMS in cerebral flow in the right dorsolateral prefrontal cortex (DLPFC) have found positive results (16-19) and, given the similarity between these two techniques, it can be assumed that the results would be similar using tDCS.

The aim of this study is to present a clinical trial study conducted in patients with PTSD caused by the "KISS nightclub fire" disaster who, being unresponsive to pharmacological therapy, underwent tDCS treatment. Symptoms of PTSD, depression and anxiety are expected to decrease and remain low after treatment and at follow-up for 60 days after intervention.

\section{Methods}

A clinical trial study was conducted from March 2015 to July 2016 at the Psychiatry Department of Santa Maria University Hospital in the Federal University of Santa Maria, Rio Grande do Sul, Brazil. The researchers followed the ethical guidelines of the 1975 Declaration of Helsinki. The research was approved by the institution's Research Ethics Committee and is registered under number 
39908114.4.0000.5346 and registered in The Brazilian Clinical Trials Registry (ReBEC) under number RBR-2qpv74b (01/07/2021). All subjects were informed of the objectives of the study and consented to participate by signing the informed consent form. The study follows the Transparent Reporting of Evaluations with Nonrandomized Designs (TREND) statement.

Patients were recruited from the Integrated Center for the Attention of Accident Victims of the Psychiatry Department of Santa Maria University Hospital, which is responsible for the attendance of patients of the "KISS nightclub fire" disaster. The inclusion criteria used for the study were: patients over 18 years of age diagnosed with PTSD without complete remission of symptoms; and maintenance of the pharmacological schedule unchanged in the three weeks preceding the beginning of the proposed treatment and during the proposed treatment. The study exclusion criteria were: patients with psychiatric indication for hospitalization; patients with severe personality disorder; presence of neurological diseases or neoplasms in activity; presence of neurodegenerative diseases; patients with metallic implants; and pregnant women and nursing mothers.

\section{Study protocol}

Neuroimaging studies suggest that the pathogenesis of PTSD may be related to changes in cortical excitability $(20,21)$. Individuals exposed to trauma memories have increased cerebral flow in the right DLPFC, suggesting hyperexcitability in this region (22). Consequently, neuromodulation in this area would have the potential to rebalance brain function and return it to basal levels (17). tDCS sessions were performed with two electrodes positioned as follows: the cathode on the right DLPFC and the extracephalic anode on the contralateral deltoid muscle. The stimulations were performed once a day, each for $30 \mathrm{~min}$; a total of 10 sessions were held over 10 continuous days. In each tDCS a current of $2 \mathrm{~mA}$ was used for an area of $25 \mathrm{~cm}^{2}$ (current density of $0.08 \mathrm{~mA} / \mathrm{cm}^{2}$ ). The electric current was administered through a direct current electrostimulator (Striat Ibramed). In order to minimize possible discomfort and increase the tolerability of the procedure, the current was maintained at $1 \mathrm{~mA}$ in the initial and final $30 \mathrm{~s}$ of each session in order to scale the current increase and decrease, thus avoiding sudden changes in its intensity. The electrodes used were made of silicone and wrapped in sponges soaked in saline solution.

\section{Assessment}

All patients were assessed using four standardized scales applied at four distinct times: before tDCS (preintervention), after tDCS (post-intervention), 30 days' post-intervention and 90-days' post-intervention.

The Post-Traumatic Stress Disorder Checklist, Civilian version (PCL-C), is composed of 17 items based on the DSM-IV for the purpose of screening and to aid in diagnostic evaluation and monitoring. Each question has five possible answers, ranging from 1 (nothing) to 5 (very). It is a self-applicable scale and its total score ranges from 17 to 85 points, classified as: $28-29$, some PTSD symptoms; $30-44$, moderate to moderately high severity of PTSD symptoms; $45-85$, high severity of PTSD symptoms (23). 
The Montreal Cognitive Assessment (MoCA) is a screening tool for mild cognitive impairment. It has a short application time, and its score varies in the range $0-30$ points. Scores greater than 26 are considered normal (24).

The Hamilton Depression Rating Scale (HAM-D) is widely used in research and clinical practice to assess patients with depressive symptoms. The version with 17 items was adopted for this trial, which is the most commonly used version (25). The scores are classified as: $0-7$, normal; $8-16$, mild depression; $17-$ 23 , moderate depression; and $>24$, severe depression (26).

The Hamilton Anxiety Rating Scale (HAM-A) consists of 14 items, with scores in the range $0-56$ points (27). Each item is evaluated from 0 (none) to 4 (maximum) points. The intensity of anxious symptoms is classified as: $<17$, mild severity; $18-24$, mild to moderate severity; and $25-30$, moderate to severe severity (28).

\section{Statistical analysis}

Statistical analysis was performed using SPSS software, version 18.0 (SPSS Inc., IBM Corporation, Armonk, NY). The Kolmogorov-Smirnov test was applied to verify the distribution of variables. Normal quantitative variables were expressed as mean and standard deviation. Qualitative variables were expressed by their absolute and relative frequencies. To examine possible differences between repeated measures of the PCL-C, MoCA, HAM-D and HAM-A for the follow-up of subjects, generalized estimating equation (GEE) analysis with the Wald chi-square test was used. To verify the exact stratum where the difference was found, the Bonferroni post-hoc test was used. All statistical analyses performed were considered significant when the bicaudal $p$ value was $<0.05$.

\section{Results}

Data from 145 subjects were initially screened. After applying the inclusion and exclusion criteria, eight subjects were analysed (flow diagram - figure 1). The characteristics of the participants are shown in Table 1. Most of the subjects were female (87.5\%) and the mean age of the studied population was 30.88 \pm 7.74 years (mean \pm standard deviation), ranging from 23 to 44 years. 
Table 1

Population characteristics

\begin{tabular}{|c|c|}
\hline Variables & $n(\%)$ \\
\hline \multicolumn{2}{|l|}{ Marital status } \\
\hline Married/stable union & $4(50.0)$ \\
\hline Single & $3(37.5)$ \\
\hline Widow & $1(12.5)$ \\
\hline \multicolumn{2}{|l|}{ Children } \\
\hline No & $5(62.5)$ \\
\hline Yes & $3(37.5)$ \\
\hline \multicolumn{2}{|l|}{ Ethnicity } \\
\hline Brown & $2(25.0)$ \\
\hline White & $6(75.0)$ \\
\hline \multicolumn{2}{|l|}{ Religion } \\
\hline Catholic & $4(50.0)$ \\
\hline Spiritist & $2(25.0)$ \\
\hline No religion & $2(25.0)$ \\
\hline \multicolumn{2}{|l|}{ Employment } \\
\hline Away from work & $1(12.5)$ \\
\hline Worker & $4(50.0)$ \\
\hline Student & $3(37.5)$ \\
\hline \multicolumn{2}{|l|}{ Education } \\
\hline Completed college & $3(37.5)$ \\
\hline Completed high school & $3(37.5)$ \\
\hline Incompleted college & $2(25.0)$ \\
\hline \multicolumn{2}{|c|}{ Family income (minimum wages) } \\
\hline $1-2$ & $1(14.3)$ \\
\hline 3 & $1(14.3)$ \\
\hline $3-5$ & $3(42.8)$ \\
\hline $6-10$ & $2(28.6)$ \\
\hline
\end{tabular}

Page 6/18 


\begin{tabular}{|cc|}
\hline Variables & $\boldsymbol{n}(\%)$ \\
\hline Comorbidities & \\
\hline No & $3(37.5)$ \\
Yes & $5(62.5)$ \\
\hline Use of medication & \\
\hline No & $0(0.0)$ \\
\hline Yes & $8(100.0)$ \\
\hline
\end{tabular}

GEE analyses were performed to evaluate possible differences from the repeated measures realized at different assessment times within each scale (PCL-C, MoCA, HAM-D and HAM-A). The Wald chi-square test and the respective $p$ value were significant for all the scales (PCL-C: $\chi^{2}=12.139, p=0.007 ;$ MoCA: $\chi^{2}$ $=12.122, p=0.007$; HAM-D: $\chi^{2}=29.146, p<0.001 ;$ HAM-A: $\left.\chi^{2}=57.989, p<0.001\right)$. This finding demonstrates that all the scales have statistically significant differences. The Bonferroni post-hoc test was used to emphasize between which measurements the significant difference was found. The results of these analyses are presented below.

It was observed that immediately after application of tDCS there was a large decrease in the scales of depression (HAM-D), anxiety (HAM-A) and PTSD (PCL-C). More details are shown in Table 2. Subjects with moderate depression diagnoses on the HAM-D scale had an average reduction of $60 \%$ in scale values after tDCS, indicating a normal diagnosis on this scale. For symptoms of anxiety measured by the HAM-A scale, patients with a diagnosis of moderate to severe anxiety presented a reduction of $54.39 \%$ after tDCS, indicating mild symptoms of anxiety. There was a $20 \%$ decrease in the values of the PCL-C scale: patients with high severity symptoms of PTSD were assessed as having moderate to moderately high severity symptoms after tDCS. Evaluation of the MoCA scale showed that there was no cognitive impairment either before or after tDCS, with patients according to this scale assessment considered normal.

Table 2

Immediate evaluation after transcranial direct current stimulation (tDCS)

\begin{tabular}{|llll|}
\hline Variables & Pre-intervention & Post-intervention & Bonferroni $p$ value \\
\hline PCL-C (mean \pm SD) & $50.38 \pm 5.55$ & $40.00 \pm 5.56$ & $<0.001$ \\
\hline MoCA (mean \pm SD) & $27.00 \pm 0.98$ & $27.50 \pm 0.66$ & 1.00 \\
\hline HAM-D (mean \pm SD) & $17.50 \pm 2.27$ & $7.86 \pm 0.99$ & $<0.001$ \\
\hline HAM-A (mean \pm SD) & $25.50 \pm 3.69$ & $11.63 \pm 1.79$ & $<0.001$ \\
\hline $\begin{array}{l}\text { PCL-C = Post-Traumatic Stress Disorder Checklist, Civilian version; SD = standard deviation; MoCA } \\
\text { Montreal Cognitive Assessment; HAM-D = Hamilton Depression Rating Scale; HAM-A = Hamilton } \\
\text { Anxiety Rating Scale. }\end{array}$ & \\
\hline
\end{tabular}


Although the scales showed an increase in their values from post-intervention to the 30-day' postintervention assessment (Table 3), these increases were not statistically significant, demonstrating that there was no significant involution of the improvements obtained with the treatment. Therefore, the effects of the treatment remained 30 days after the intervention.

Table 3

Post-intervention evaluation compared to 30-day evaluation

\begin{tabular}{|c|c|c|c|}
\hline Variables & Post-intervention & 30-Day post-intervention & Bonferroni $p$ value \\
\hline PCL-C (mean \pm SD $)$ & $40.00 \pm 5.56$ & $42.63 \pm 5.69$ & 0.248 \\
\hline MoCA $($ mean \pm SD $)$ & $27.50 \pm 0.66$ & $28.50 \pm 0.59$ & 0.356 \\
\hline HAM-D (mean \pm SD) & $7.86 \pm 0.99$ & $10.71 \pm 2.11$ & 1.00 \\
\hline HAM-A (mean \pm SD) & $11.63 \pm 1.79$ & $15.57 \pm 3.28$ & 1.000 \\
\hline
\end{tabular}

Comparing pre-intervention and 30-day post-intervention values (Table 4), there was a statistically significant decrease in the PCL-C values and a 5.5\% improvement of cognitive function in the MoCA scale.

Table 4

Pre-intervention evaluation compared to 30-day evaluation

\begin{tabular}{|llll|}
\hline Variables & Pre-intervention & 30-Day post-intervention & Bonferroni $\boldsymbol{p}$ value \\
\hline PCL-C (mean \pm SD) & $50.38 \pm 5.55$ & $42.63 \pm 5.69$ & 0.025 \\
\hline MoCA (mean \pm SD) & $27.00 \pm 0.98$ & $28.50 \pm 0.59$ & 0.028 \\
\hline HAM-D (mean \pm SD) & $17.50 \pm 2.27$ & $10.71 \pm 2.11$ & 0.176 \\
HAM-A (mean \pm SD) & $25.50 \pm 3.69$ & $15.57 \pm 3.28$ & 0.560 \\
\hline $\begin{array}{l}\text { PCL-C = Post-Traumatic Stress Disorder Checklist, Civilian version; SD = standard deviation; MoCA } \\
\text { Montreal Cognitive Assessment; HAM-D = Hamilton Depression Rating Scale; HAM-A = Hamilton } \\
\text { Anxiety Rating Scale. }\end{array}$
\end{tabular}

The last evaluation 90 days after the intervention (Table 5) showed that the PCL-C and MoCA scale values remained low, but compared with pre-intervention the findings were not significant. With regard to depression and anxiety, there were significant improvements in values: patients diagnosed with moderate depression pre-intervention were diagnosed with mild depression at the end of 90 days; and patients diagnosed with moderate to severe anxiety symptoms pre-intervention were diagnosed with mild anxiety symptoms after 90 days. 
Table 5

Pre-intervention evaluation compared to 90-day evaluation

\begin{tabular}{|llll|}
\hline Variables & Pre-intervention & 90-Day post-intervention & Bonferroni $p$ value \\
\hline PCL-C (mean \pm SD) & $50.38 \pm 5.55$ & $37.8 \pm 7.16$ & 0.157 \\
\hline MoCA (mean \pm SD) & $27.00 \pm 0.98$ & $28.00 \pm 1.17$ & 0.219 \\
\hline HAM-D (mean \pm SD) & $17.50 \pm 2.27$ & $8.25 \pm 1.95$ & 0.006 \\
\hline HAM-A (mean \pm SD) & $25.50 \pm 3.69$ & $14.25 \pm 1.14$ & 0.028 \\
\hline $\begin{array}{l}\text { PCL-C = Post-Traumatic Stress Disorder Checklist, Civilian version; SD = standard deviation; MoCA } \\
\text { Montreal Cognitive Assessment; HAM-D = Hamilton Depression Rating Scale; HAM-A = Hamilton } \\
\text { Anxiety Rating Scale. }\end{array}$ & \\
\hline
\end{tabular}

\section{Discussion}

All patients undergoing experimental treatment with tDCS showed improvement in PTSD symptoms after the intervention and these positive effects were maintained after 30 days of intervention. Also, but in a lesser proportion, maintained improvement in relation to symptoms of depression and anxiety at the end of 90 days was observed.

Only minor and brief adverse effects have been reported: local hyperemia, pruritus, mild discomfort and headache. Local hyperemia ceased spontaneously within 1 hour after the end of the stimulus, itching and discomfort was reduced or ceased with extra application of saline solution on the sponges and headache ceased spontaneously within 24 hours on taking common analgesics. These data help to reinforce the evidence that tDCS is a safe technique for non-invasive neuromodulation $(12,13)$.

In our study, a $20 \%$ reduction in the symptoms of refractory PTSD was observed after treatment. This improvement was seen immediately after the tenth neuromodulation session and was present throughout the first month after treatment. Although the improvement continued, it was not stable over time and there was some deterioration in the positive effects over the period. However, when compared to the levels pre-intervention, the positive effects after 1 month of treatment were still statistically significant. The gradual loss of this improvement is compatible with the already known transitory effect of the neuroplastic changes induced by tDCS. One of the pioneering articles with rTMS was carried out in the 1980s by McCann et al. (18). Patients received low-frequency stimuli in DLPFC and showed an improvement in PTSD symptoms that lasted for the entire first month after treatment. Positive results were also found by Grisaru et al. (29), with individuals showing improvement in their condition for a week after applying only one stimulation session. Cohen et al. (30) conducted a clinical trial with 24 patients, dividing them into groups subjected to low-frequency, high-frequency and simulated stimuli. These authors obtained positive results in the symptoms of PTSD and anxiety for patients undergoing treatment with high frequencies compared to the other groups, as we found in our study. Another similarity is that 10 DLPFC stimulation sessions were carried out over the course of 2 weeks. 
Corroborating the importance of DLPFC neuromodulation in the treatment of PTSD, Boggio et al. (31) demonstrated that stimulation with high frequencies $(20 \mathrm{~Hz})$ in both hemispheres caused an improvement in symptoms, with these effects being more evident in the right cortex. In addition, stimulus on the right DLPFC caused an improvement in anxiety symptoms and cognition, as in our study. Watts et al. (19) also evaluated the effects of rTMS on DLPFC and observed improvement in PTSD symptoms in the group submitted to the active stimulus as opposed to the simulated stimulus, but unlike the protocol used by Boggio et al. (31) the sessions were carried out with low-frequency stimuli $(1 \mathrm{~Hz})$. In a similar study there was also an improvement in PTSD (16). These findings corroborate what we found in our research, due to the similarity between the cathodic stimuli of tDCS and the low frequencies in rTMS. There are several examples in the literature demonstrating the benefits of neuromodulation of the right prefrontal cortex and its repercussions both on the symptoms of PTSD and the frequent comorbidities of this disorder. The results found in our study corroborate those observed in other studies, reinforcing the importance of the right DLPFC. A peculiarity of our research was that, unlike most studies, which applied excitatory stimuli, we applied inhibitory stimuli and, even so, we obtained similar positive results. There is an increasing amount of evidence suggesting the relevance of DLPFC in PTSD and neuromodulation of this region proves to be a good alternative to be added to the treatment arsenal of this disorder. However, some aspects deserve attention and should be better clarified, such as differentiating and quantifying the action of excitatory and inhibitory stimuli, as both seem to have beneficial effects. Not only should tDCS be explored as an isolated alternative but also as an adjunct treatment to psychotropic drugs or cognitive behavioral therapy. In addition, it should be analyzed whether improvement of the condition is due to improvement of PTSD itself or is an apparent improvement, a reflection of the important relief of concomitant depressive and anxious symptoms. All individuals undergoing experimental treatment in our study were refractory and had already used at least two different pharmacological regimens. They had been symptomatic patients for longer, symptoms were more severe and they responded less to conventional treatment. Therefore, the positive response to tDCS specifically in this group would have been less than expected when compared to patients with less intense symptoms, which seems to be intuitive.

The main characteristic of depressive disorder is the presence of sadness and/or anhedonia, in addition to changes in sleep and appetite, tiredness, feelings of guilt and worthlessness, cognitive impairment and ideas of death. It is a highly prevalent disease and is a frequent comorbidity in patients with PTSD (32). In our study, after the tenth session of tDCS the depressive symptoms improved by $60 \%$. This improvement was still noticeable after 1 month of treatment; however, the intensity of the improvement decreased over the period. Most studies that analyzed the effect of tDCS on depressive symptoms were carried out with unilateral assemblies applying the anodic stimulus in the left DLPFC because the hypoactivity of this area is one of the pathophysiological hypotheses of this disorder. Neuromodulation of this region has been promising, with a response comparable to the use of fluoxetine (33), and the association of tDCS with sertraline proved to be superior to the use of tDCS or sertraline alone (34). Other studies have also shown encouraging results. Boggio et al. (31), in a randomized controlled study of 40 patients and with the anode on the left, found a $40 \%$ improvement in depressive symptoms. Similar 
studies have also found positive results $(33,35)$, with a reduction of up to $60 \%$ in symptoms. However, due to the large functional differences between the cerebral hemispheres, it is impossible to compare these studies with this research. In contrast to these positive results, Loo et al. (36) randomized 64 depressed subjects resistant to treatment into active and simulated groups and found no significant difference between groups. One possible explanation for this may be the fact that fewer sessions were held (five in total). However, a clinical trial (37) that stimulated depressed patients resistant to escitalopram by taking 10 sessions also found no positive results. It is interesting to take into account that some studies that showed robust results in the reduction of depressive symptoms $(18,35)$ interrupted the use of antidepressants for up to 2 months before starting the procedure. For ethical reasons, we kept patients on standard treatment and achieved similar results. So far, there have been no unipolar assemblies used in the right prefrontal region in depressed patients. The closest have been bilateral assemblies in which the anode on the left is associated with the cathode on the right. Brunoni et al. (34), using an assembly similar to the one described, observed cognitive impairment in the right prefrontal cortex but it was the opposite of what we found in this study, in which there was an improvement in depressive and cognitive symptoms. The small number of patients in this study makes it impossible to generalize, yet all patients showed improvement in depression and none of them evolved with worsening cognition. In contrast to other studies, we observed a significant improvement in depressive symptoms without concomitant cognitive impairment. The role of the right DLPFC in depressive symptoms needs to be better understood, especially its role in the regulation of subcortical structures related to depressive symptoms. In addition, chronically ill patients are more likely to develop depressive disorder and the improvement in PTSD may have influenced the improvement in depression.

Our study showed a $54 \%$ improvement in anxiety symptoms. This improvement was present 30 days after the end of treatment, despite some decrease in these effects. Even so, this improvement remained statistically significant when compared to before treatment. Although anxiety disorders are quite prevalent, there is still little research involving tDCS in patients with these symptoms and, so far, it is limited to case reports. There is only one case report of a patient with generalized anxiety disorder refractory to treatment undergoing tDCS sessions (38). As in our study, cathodic stimulus was applied in the right DLPFC with improvement of symptoms. There is also a case report of a patient with obsessivecompulsive disorder resistant to treatment who underwent cathodic stimulus in order to decrease cortical excitability. There was a $30 \%$ reduction in the severity of the condition but stimulation was performed in the motor cortex (39). Evidence of tDCS action on anxiety disorders is still incipient. This is partly due to the fact that anxiety disorders are very heterogeneous, involving different brain areas. It is expected that with the development of larger studies, the evidence supporting the use of this modality of neuromodulation as an alternative to standard treatment will increase.

There was a gradual improvement in cognition immediately after treatment. However, this improvement was only statistically significant when the patients were reassessed after 1 month of treatment, suggesting that the beneficial effects may have progressed even after the end of the stimulations. The sphere of cognition is very heterogeneous and composed of several aspects, such as attention, vigilance, perception (visual, auditory, somatosensory), working memory, learning, decision-making, etc., located in 
different brain circuits. The involvement of DLPFC in the various aspects of neurocognition has been the subject of investigation and numerous studies in the literature have shown positive effects on cognition after anodic stimuli, mainly in the left DLPFC (40-43). Although patients undergoing experimental treatment have improved cognition, the literature shows that, unlike what happens with anodic stimuli, cathodic stimuli applied in the right and left DLPFC are related to worsening cognitive performance. Tanoue et al. (44) conducted a study to assess attention by dividing 24 patients into three groups: cathodic stimulation in the right parietal region; cathodic stimulation in the right DLPFC; and sham stimulus (simulated stimulation, performed with the device turned off). Patients undergoing cathodic stimulation showed worse performance when compared to the sham stimulus. In addition, this worsening of performance was more intense in patients stimulated in the right DLPFC. Elmer et al. (45) also observed a worsening of cognition after cathodic stimulation in the left DLPFC. Opposing these data, another study found that anodic stimulation in the right DLPFC improves learning and attention (40), corroborating the opposite effect that the cathode and anode exert. What would justify this apparent improvement in the cognitive performance of stimulated patients is the fact that the MoCA is a simple scale with a learning curve. There is a possibility that this improvement does not necessarily reflect an improvement in cognition but rather a memorization of the test by patients. Still, this scale alone is insufficient in view of the complexity of the cognitive domains, and for a more complete assessment it would be necessary to apply several neurocognitive tests together with tests of its different domains in a broader way. In addition, the MoCA is a screening scale for mild cognitive impairment, with some authors suggesting a cutoff point between 24 and 26 points. Of the eight patients studied, seven were above 26 points in all evaluations, that is, although they did not score the total 30 points they were outside the range considered for the presence of cognitive impairment. The study of cognitive processes is a complex area and the application of cathodic stimuli, which decrease cortical excitability, is not necessarily harmful, as demonstrated by Weiss and Lavidor (46) when they stimulated the parietal cortex of healthy individuals, which was corroborated by our work.

Important limiting factors of the study were the small number of participants, which makes generalizations difficult, and the absence of a control group. Individual characteristics also had a limiting effect as age, gender and shape and size of the skull can interfere in the amount of current reaching the brain and also in the correct positioning of the electrodes. In addition, all patients were using one or more psychotropic drugs and therefore were subject to interference from these drugs in cortical excitability. It has been shown that the use of psychotropic drugs influences the rate of neuronal firing and may be related to longer lasting effects or decreased effects of tDCS (47). However, it is a unique study carried out in a population of young adults exposed to the same traumatic event, which was unique in our country and in the world, and the study used validated assessment tools for depression, anxiety, cognition and PTSD.

\section{Conclusion}

Our study found that patients with refractory PTSD, after adjuvant treatment with tDCS, showed improvement of $20 \%$ in the central symptoms of PTSD, in addition to an improvement of $60 \%$ in 
depressive symptoms and $54 \%$ in anxious symptoms. Despite a decrease in these effects over time, this improvement was maintained throughout the first month after treatment. The improvement in cognition occurred slowly and progressively, being statistically significant at the end of the first month of treatment. There was no development of important side effects. Non-invasive neuromodulation techniques, especially tDCS, have been shown to be safe and promising in the treatment of neuropsychiatric pathologies and can be an alternative for the treatment of refractory disorders, either as a monotherapy or as a treatment enhancement strategy. They can also be an option for patients who do not want or do not tolerate pharmacological management.

There are multiple conditions that can influence the outcome of the procedure and should be studied in future research. The most important of these in relation to the stimulation protocol itself are the size of the stimulated area, the duration of the stimulus, the intensity of the current and the number of days of stimulation. The response of specific brain regions to tDCS should be further explored because different regions submitted to the same stimulus may not have similar effects.

\section{Abbreviations}

DLPFC Right dorsolateral prefrontal cortex

GEE Generalized estimating equation

HAM-A Hamilton Anxiety Rating Scale

HAM-D Hamilton Depression Rating Scale

MoCA Montreal Cognitive Assessment

PCL-C Post-Traumatic Stress Disorder Checklist, Civilian version

PTSD Post-traumatic stress disorder

ReBEC Brazilian Clinical Trials Registry

rTMS Repetitive transcranial magnetic stimulation

tDCS transcranial stimulation by continuous current

TREND Transparent Reporting of Evaluations with Nonrandomized Designs

\section{Declarations}

AUTHOR AGREEMENT AND SUBMISSION DECLARATION: All authors have seen and approved the final version of the manuscript being submitted. They warrant that the article is the authors' original work, hasn't received prior publication and isn't under consideration for publication elsewhere. 
DECLARATION OF INTEREST: None.

FUNDING SOURCE DELCARATION: No funding or grants or equipment were provided for the study from any source. No financial benefits were received by the authors. This study was not published in this or any other form (abstracts or other abbreviated forms).

DETAILS OF ETHICS APPROVAL: The study was submitted to the institutional review board (IRB) of our university (Federal University of Santa Maria, Santa Maria, Rio Grande do Sul, Brazil) and was approved under the number 39908114.4.0000.5346. The informed consent was also approved by the IRB, as well as the confidentiality statement. After approval, the study began, and all the participants signed the informed consent.

CONSENT FOR PUBLICATION: The manuscript does not contains any individual person's data in any form (including any individual details, images, or videos), that require obtaining consent for publication.

ACKNOWLEDGEMENTS: none.

COMPETING INTERESTS: none.

\section{AUTHORS' CONTRIBUTIONS:}

- KASM: data collection;

- KASM, ABMC, BCY and TAR: writing the paper, revising the paper;

- TAR: data analysis;

- TAR and ABMC: orientation.

AVAILABILITY OF DATA AND MATERIALS: the datasets generated and/or analysed during the current study are available in the Mendeley Data repository, http://dx.doi.org/10.17632/3y64b8fdnh.1

\section{References}

1. Hori H, Kim Y. Inflammation and post-traumatic stress disorder. Psychiatry Clin Neurosci. 2019;73(4):143-53.

2. Hunter SR. Drawing soldiers out of post-traumatic stress disorder. Mil Med Res. 2019;6(1):5.

3. Pitman RK, Rasmusson AM, Koenen KC, Shin LM, Orr SP, Gilbertson MW, et al. Biological studies of post-traumatic stress disorder. Nat Rev Neurosci. 2012;13(11):769-87.

4. Qi W, Gevonden M, Shalev A. Prevention of Post-Traumatic Stress Disorder After Trauma: Current Evidence and Future Directions. Curr Psychiatry Rep. 2016;18(2):20.

5. Javidi H, Yadollahie M. Post-traumatic Stress Disorder. Int J Occup Environ Med. 2012;3(1):2-9.

6. Kessler RC, Sonnega A, Bromet E, Hughes M, Nelson CB. Posttraumatic stress disorder in the National Comorbidity Survey. Arch Gen Psychiatry. 1995;52(12):1048-60. 
7. McFarlane AC, Williamson P, Barton CA. The impact of traumatic stressors in civilian occupational settings. J Public Health Policy. 2009;30(3):311-27.

8. Group TMOPSDW. VA/DOD CLINICAL PRACTICE GUIDELINE FOR THE MANAGEMENT OF POSTTRAUMATIC STRESS DISORDER AND ACUTE STRESS DISORDER In: Affairs DoV, Defense Do, editors. Washington, D.C2017.

9. Kirkpatrick HA, Heller GM. Post-traumatic stress disorder: theory and treatment update. Int J Psychiatry Med. 2014;47(4):337-46.

10. Cooper J, Carty J, Creamer M. Pharmacotherapy for posttraumatic stress disorder: empirical review and clinical recommendations. Aust N Z J Psychiatry. 2005;39(8):674-82.

11. Berger W, Mendlowicz MV, Marques-Portella C, Kinrys G, Fontenelle LF, Marmar CR, et al. Pharmacologic alternatives to antidepressants in posttraumatic stress disorder: a systematic review. Prog Neuropsychopharmacol Biol Psychiatry. 2009;33(2):169-80.

12. Nitsche MA, Cohen LG, Wassermann EM, Priori A, Lang N, Antal A, et al. Transcranial direct current stimulation: State of the art 2008. Brain Stimul. 2008;1(3):206-23.

13. Novakovic V, Sher L, Lapidus KA, Mindes J, J AG, Yehuda R. Brain stimulation in posttraumatic stress disorder. Eur J Psychotraumatol. 2011;2.

14. Brunoni AR, Nitsche MA, Bolognini N, Bikson M, Wagner $T$, Merabet $L$, et al. Clinical research with transcranial direct current stimulation (tDCS): challenges and future directions. Brain Stimul. 2012;5(3):175-95.

15. Polania R, Nitsche MA, Paulus W. Modulating functional connectivity patterns and topological functional organization of the human brain with transcranial direct current stimulation. Hum Brain Mapp. 2011;32(8):1236-49.

16. Nam DH, Pae CU, Chae JH. Low-frequency, Repetitive Transcranial Magnetic Stimulation for the Treatment of Patients with Posttraumatic Stress Disorder: a Double-blind, Sham-controlled Study. Clin Psychopharmacol Neurosci. 2013;11(2):96-102.

17. Osuch EA, Benson BE, Luckenbaugh DA, Geraci M, Post RM, McCann U. Repetitive TMS combined with exposure therapy for PTSD: a preliminary study. J Anxiety Disord. 2009;23(1):54-9.

18. McCann UD, Kimbrell TA, Morgan CM, Anderson T, Geraci M, Benson BE, et al. Repetitive transcranial magnetic stimulation for posttraumatic stress disorder. Arch Gen Psychiatry. 1998;55(3):276-9.

19. Watts BV, Landon B, Groft A, Young-Xu Y. A sham controlled study of repetitive transcranial magnetic stimulation for posttraumatic stress disorder. Brain Stimul. 2012;5(1):38-43.

20. Shin LM, Kosslyn SM, McNally RJ, Alpert NM, Thompson WL, Rauch SL, et al. Visual imagery and perception in posttraumatic stress disorder. A positron emission tomographic investigation. Arch Gen Psychiatry. 1997;54(3):233-41.

21. Shin LM, McNally RJ, Kosslyn SM, Thompson WL, Rauch SL, Alpert NM, et al. A positron emission tomographic study of symptom provocation in PTSD. Ann N Y Acad Sci. 1997;821:521-3. 
22. Rauch SL, van der Kolk BA, Fisler RE, Alpert NM, Orr SP, Savage CR, et al. A symptom provocation study of posttraumatic stress disorder using positron emission tomography and script-driven imagery. Arch Gen Psychiatry. 1996;53(5):380-7.

23. Wilkins KC, Lang AJ, Norman SB. Synthesis of the psychometric properties of the PTSD checklist (PCL) military, civilian, and specific versions. Depress Anxiety. 2011;28(7):596-606.

24. Nasreddine ZS, Phillips NA, Bedirian V, Charbonneau S, Whitehead V, Collin I, et al. The Montreal Cognitive Assessment, MoCA: a brief screening tool for mild cognitive impairment. J Am Geriatr Soc. 2005;53(4):695-9.

25. Freire MÃv, Figueiredo VLcMd, Gomide A, Jansen K, Silva RAd, MagalhÃ£es PVdS, et al. Escala Hamilton: estudo das características psicométricas em uma amostra do sul do Brasil. Jornal Brasileiro de Psiquiatria. 2014;63:281-9.

26. Zimmerman M, Martinez JH, Young D, Chelminski I, Dalrymple K. Severity classification on the Hamilton Depression Rating Scale. J Affect Disord. 2013;150(2):384-8.

27. Hamilton M. The assessment of anxiety states by rating. Br J Med Psychol. 1959;32(1):50-5.

28. Thompson E. Hamilton Rating Scale for Anxiety (HAM-A). Occup Med (Lond). 2015;65(7):601.

29. Grisaru N, Amir M, Cohen H, Kaplan Z. Effect of transcranial magnetic stimulation in posttraumatic stress disorder: a preliminary study. Biol Psychiatry. 1998;44(1):52-5.

30. Cohen H, Kaplan Z, Kotler M, Kouperman I, Moisa R, Grisaru N. Repetitive transcranial magnetic stimulation of the right dorsolateral prefrontal cortex in posttraumatic stress disorder: a double-blind, placebo-controlled study. Am J Psychiatry. 2004;161(3):515-24.

31. Boggio PS, Rigonatti SP, Ribeiro RB, Myczkowski ML, Nitsche MA, Pascual-Leone A, et al. A randomized, double-blind clinical trial on the efficacy of cortical direct current stimulation for the treatment of major depression. Int J Neuropsychopharmacol. 2008;11(2):249-54.

32. Association AP. Transtornos Relacionados a Trauma e a Estressores. In: Association AP, editor. Manual diagnóstico e estatístico de transtornos mentais DSM-5. 5 ed. Porto Alegre: Artmed; 2014. p. 265.

33. Rigonatti SP, Boggio PS, Myczkowski ML, Otta E, Fiquer JT, Ribeiro RB, et al. Transcranial direct stimulation and fluoxetine for the treatment of depression. Eur Psychiatry. 2008;23(1):74-6.

34. Brunoni AR, Valiengo L, Baccaro A, Zanao TA, de Oliveira JF, Goulart A, et al. The sertraline vs. electrical current therapy for treating depression clinical study: results from a factorial, randomized, controlled trial. JAMA Psychiatry. 2013;70(4):383-91.

35. Fregni F, Boggio PS, Nitsche MA, Marcolin MA, Rigonatti SP, Pascual-Leone A. Treatment of major depression with transcranial direct current stimulation. Bipolar Disord. 2006;8(2):203-4.

36. Loo CK, Alonzo A, Martin D, Mitchell PB, Galvez V, Sachdev P. Transcranial direct current stimulation for depression: 3-week, randomised, sham-controlled trial. Br J Psychiatry. 2012;200(1):52-9.

37. Bennabi D, Nicolier M, Monnin J, Tio G, Pazart L, Vandel P, et al. Pilot study of feasibility of the effect of treatment with tDCS in patients suffering from treatment-resistant depression treated with 
escitalopram. Clin Neurophysiol. 2015;126(6):1185-9.

38. Shiozawa P, Leiva AP, Castro CD, da Silva ME, Cordeiro Q, Fregni F, et al. Transcranial direct current stimulation for generalized anxiety disorder: a case study. Biol Psychiatry. 2014;75(11):e17-8.

39. D'Urso G, Brunoni AR, Anastasia A, Micillo M, de Bartolomeis A, Mantovani A. Polarity-dependent effects of transcranial direct current stimulation in obsessive-compulsive disorder. Neurocase. 2016;22(1):60-4.

40. Coffman BA, Trumbo MC, Clark VP. Enhancement of object detection with transcranial direct current stimulation is associated with increased attention. BMC Neurosci. 2012;13:108.

41. Clarke PJ, Browning M, Hammond G, Notebaert L, MacLeod C. The causal role of the dorsolateral prefrontal cortex in the modification of attentional bias: evidence from transcranial direct current stimulation. Biol Psychiatry. 2014;76(12):946-52.

42. Fregni F, Boggio PS, Nitsche M, Bermpohl F, Antal A, Feredoes E, et al. Anodal transcranial direct current stimulation of prefrontal cortex enhances working memory. Exp Brain Res. 2005;166(1):2330.

43. Zaehle T, Sandmann P, Thorne JD, Jancke L, Herrmann CS. Transcranial direct current stimulation of the prefrontal cortex modulates working memory performance: combined behavioural and electrophysiological evidence. BMC Neurosci. 2011;12:2.

44. Tanoue RT, Jones KT, Peterson DJ, Berryhill ME. Differential frontal involvement in shifts of internal and perceptual attention. Brain Stimul. 2013;6(4):675-82.

45. Elmer S, Burkard M, Renz B, Meyer M, Jancke L. Direct current induced short-term modulation of the left dorsolateral prefrontal cortex while learning auditory presented nouns. Behav Brain Funct. 2009;5:29.

46. Weiss M, Lavidor M. When less is more: evidence for a facilitative cathodal tDCS effect in attentional abilities. J Cogn Neurosci. 2012;24(9):1826-33.

47. Liebetanz D, Nitsche MA, Tergau F, Paulus W. Pharmacological approach to the mechanisms of transcranial DC-stimulation-induced after-effects of human motor cortex excitability. Brain. 2002;125(Pt 10):2238-47.

\section{Figures}




\section{Enrollment}

Excluded $(n=84)$

- Discharge from treatment $(n=2)$

- Never attended the scheduled appointment $(n=11)$

- Abandoned treatment $(n=9)$

- Other diagnoses $(n=62)$

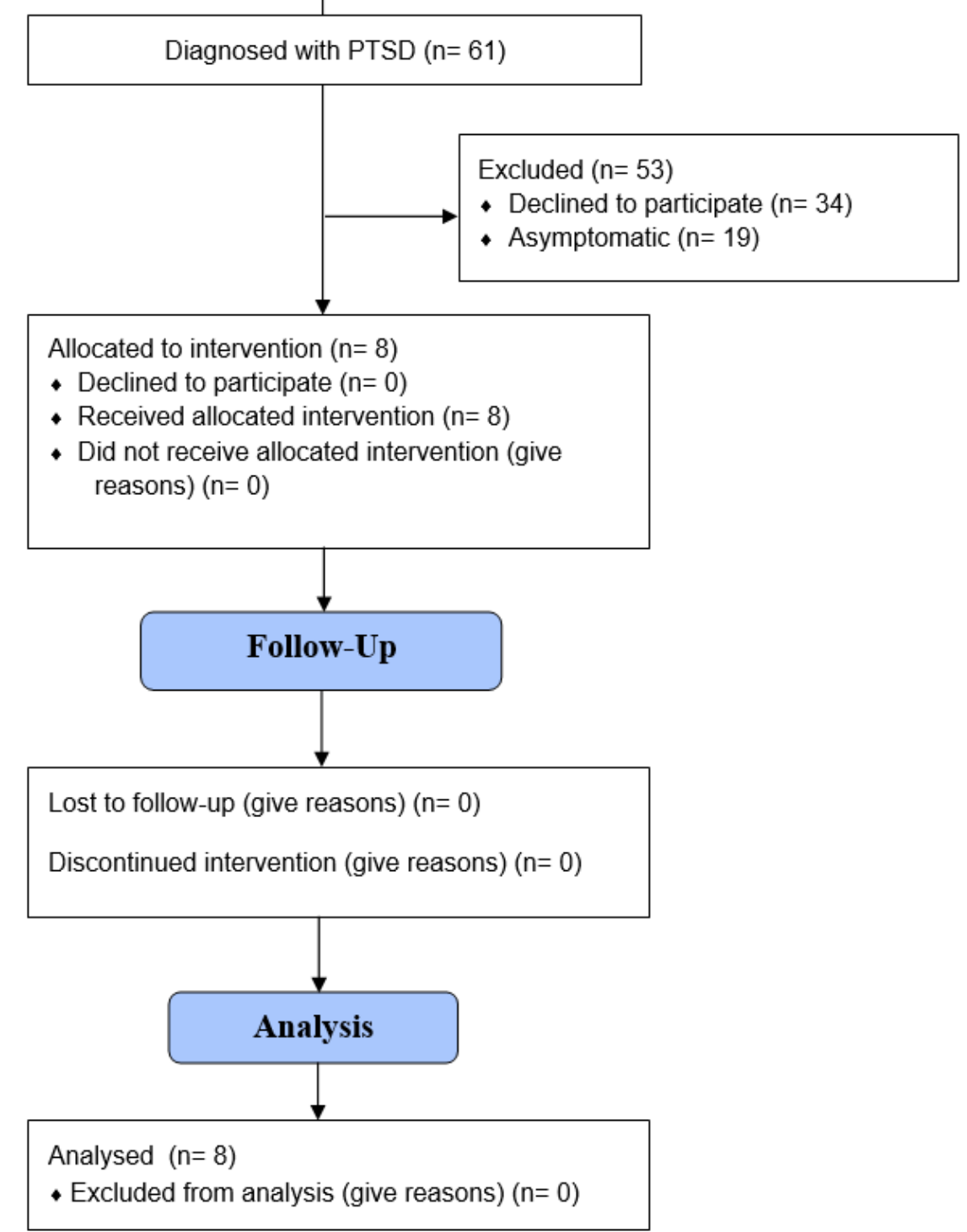

\section{Figure 1}

Study flow diagram 